\title{
What Makes Paris Look like Paris?
}

\author{
Carl Doersch ${ }^{1} \quad$ Saurabh Singh $^{1} \quad$ Abhinav Gupta $^{1} \quad$ Josef Sivic $^{2} \quad$ Alexei A. Efros $^{1,2}$ \\ ${ }^{1}$ Carnegie Mellon University $\quad{ }^{2}$ INRIA / Ecole Normale Supérieure, Paris
}
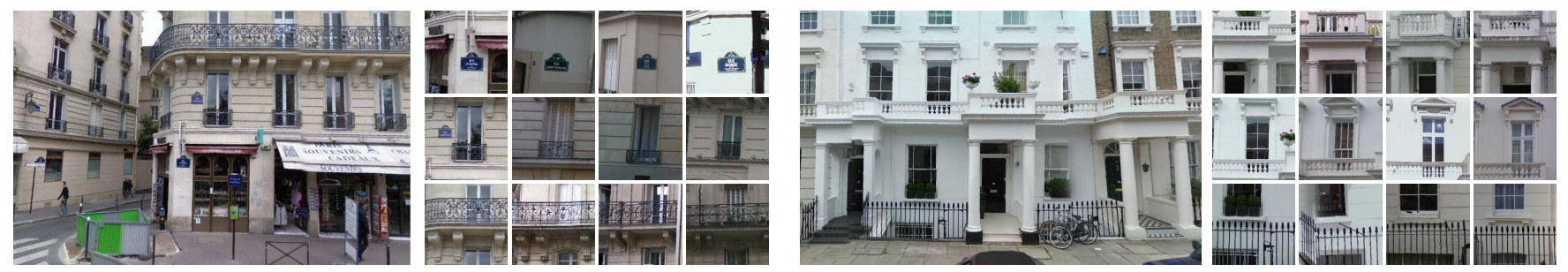

Figure 1: These two photos might seem nondescript, but each contains hints about which city it might belong to. Given a large image database of a given city, our algorithm is able to automatically discover the geographically-informative elements (patch clusters to the right of each photo) that help in capturing its "look and feel". On the left, the emblematic street sign, a balustrade window, and the balcony support are all very indicative of Paris, while on the right, the neoclassical columned entryway sporting a balcony, a Victorian window, and, of course, the cast iron railing are very much features of London.

\section{Abstract}

Given a large repository of geotagged imagery, we seek to automatically find visual elements, e.g. windows, balconies, and street signs, that are most distinctive for a certain geo-spatial area, for example the city of Paris. This is a tremendously difficult task as the visual features distinguishing architectural elements of different places can be very subtle. In addition, we face a hard search problem: given all possible patches in all images, which of them are both frequently occurring and geographically informative? To address these issues, we propose to use a discriminative clustering approach able to take into account the weak geographic supervision. We show that geographically representative image elements can be discovered automatically from Google Street View imagery in a discriminative manner. We demonstrate that these elements are visually interpretable and perceptually geo-informative. The discovered visual elements can also support a variety of computational geography tasks, such as mapping architectural correspondences and influences within and across cities, finding representative elements at different geo-spatial scales, and geographically-informed image retrieval.

CR Categories: I.3.m [Computer Graphics]: MiscellaneousApplication I.4.10 [Image Processing and Computer Vision]: Image Representation-Statistical

Keywords: data mining, visual summarization, reference art, big data, computational geography, visual perception

Links: $\odot$ DL

\section{Introduction}

Consider the two photographs in Figure 1, both downloaded from Google Street View. One comes from Paris, the other one from London. Can you tell which is which? Surprisingly, even for these nondescript street scenes, people who have been to Europe tend to do quite well on this task. In an informal survey, we presented 11 subjects with 100 random Street View images of which $50 \%$ were from Paris, and the rest from eleven other cities. We instructed the subjects (who have all been to Paris) to try and ignore any text in the photos, and collected their binary forced-choice responses (Paris / Not Paris). On average, subjects were correct $79 \%$ of the time (std $=6.3$ ), with chance at $50 \%$ (when allowed to scrutinize the text, performance for some subjects went up as high as $90 \%$ ). What this suggests is that people are remarkably sensitive to the geographically-informative features within the visual environment. But what are those features? In informal debriefings, our subjects suggested that for most images, a few localized, distinctive elements "immediately gave it away". E.g. for Paris, things like windows with railings, the particular style of balconies, the distinctive doorways, the traditional blue/green/white street signs, etc. were particularly helpful. Finding those features can be difficult though, since every image can contain more than 25, 000 candidate patches, and only a tiny fraction will be truly distinctive.

In this work, we want to find such local geo-informative features automatically, directly from a large database of photographs from a particular place, such as a city. Specifically, given tens of thousands of geo-localized images of some geographic region $R$, we aim to find a few hundred visual elements that are both: 1) repeating, i.e. they occur often in $R$, and 2) geographically discriminative, i.e. they occur much more often in $R$ than in $R^{C}$. Figure 1 shows sample output of our algorithm: for each photograph we show three of the most geo-informative visual elements that were automatically discovered. For the Paris scene (left), the street sign, the window with railings, and the balcony support are all flagged as informative.

But why is this topic important for modern computer graphics? 1) Scientifically, the goal of understanding which visual elements are fundamental to our perception of a complex visual concept, such as a place, is an interesting and useful one. Our paper shares this motivation with a number of other recent works that don't actually synthesize new visual imagery, but rather propose ways of finding and visualizing existing image data in better ways, be it selecting candid portraits from a video stream [Fiss et al. 2011], summarizing 
a scene from photo collections [Simon et al. 2007], finding iconic images of an object [Berg and Berg 2009], etc. 2) More practically, one possible future application of the ideas presented here might be to help CG modelers by generating so-called "reference art" for a city. For instance, when modeling Paris for PIXAR's Ratatouille, the co-director Jan Pinkava faced exactly this problem: "The basic question for us was: "what would Paris look like as a model of Paris?', that is, what are the main things that give the city its unique look?" [Paik 2006]. Their solution was to "run around Paris for a week like mad tourists, just looking at things, talking about them, and taking lots of pictures" not just of the Eiffel Tower but of the many stylistic Paris details, such as signs, doors etc. [Paik 2006](see photos on pp.120-121). But if going "on location" is not feasible, our approach could serve as basis for a detail-centric reference art retriever, which would let artists focus their attention on the most statistically significant stylistic elements of the city. 3) And finally, more philosophically, our ultimate goal is to provide a stylistic narrative for a visual experience of a place. Such narrative, once established, can be related to others in a kind of geo-cultural visual reference graph, highlighting similarities and differences between regions. E.g. one could imagine finding a visual appearance "trail" from Greece, through Italy and Spain and into Latin America. In this work, we only take the first steps in this direction - connecting visual appearance across cities, finding similarities within a continent, and differences between neighborhoods. But we hope that our work might act as a catalyst for research in this new area, which might be called computational geo-cultural modeling.

\section{Prior Work}

In the field of architectural history, descriptions of urban and regional architectural styles and their elements are well established, e.g. [Loyer 1988; Sutcliffe 1996]. Such local elements and rules for combining them have been used in computer systems for procedural modeling of architecture to generate 3D models of entire cities in an astonishing level of detail, e.g. [Mueller et al. 2006], or to parse images of facades, e.g. [Teboul et al. 2010]. However, such systems require significant manual effort from an expert to specify the appropriate elements and rules for each architectural style.

At the other end of the spectrum, data-driven approaches have been leveraging the huge datasets of geotagged images that have recently become available online. For example, Crandall et al. [2009] use the GPS locations of 35 thousand consumer photos from Flickr to plot photographer-defined frequency maps of cities and countries, while Kalogerakis et al. [2009] use the locations and relative time-stamps of photos of the same photographer to model worldwide human travel priors. Geo-tagged datasets have also been used for place recognition [Schindler et al. 2007; Knopp et al. 2010; Chen et al. 2011] including famous landmarks [Li et al. 2008; Li et al. 2009; Zheng et al. 2009]. Our work is particularly related to [Schindler et al. 2007; Knopp et al. 2010], where geotags are also used as a supervisory signal to find sets of image features discriminative for a particular place. While these approaches can work very well, their image features typically cannot generalize beyond matching specific buildings imaged from different viewpoints. Alternatively, global image representations from scene recognition, such as GIST descriptor [Oliva and Torralba 2006] have been used for geo-localization of generic scenes on the global Earth scale [Hays and Efros 2008; Kalogerakis et al. 2009]. There, too, reasonable recognition performance has been achieved, but the use of global descriptors makes it hard for a human to interpret why a given image gets assigned to a certain location.

Finally, our paper is related to a line of work on unsupervised object discovery [Russell et al. 2006; Chum et al. 2009; Karlinsky et al. 2009; Lee and Grauman 2009; Singh et al. 2012] (and espe- cially [Quack et al. 2008], who also deal with mining geo-tagged image data). Such methods attempt to explicitly discover features or objects which occur frequently in many images and are also useful as human-interpretable elements of visual representation. But being unsupervised, these methods are limited to only discovering things that are both very common and highly visually consistent. However, adding supervision, such as by explicitly training object [Li et al. 2010] or object part detectors [Bourdev and Malik 2009], requires a labor-intensive labeling process for each object/part.

In contrast, here we propose a discovery method that is weakly constrained by location labels derived from GPS tags, and which is able to mine representative visual elements automatically from a large online image dataset. Not only are the resulting visual elements geographically discriminative (i.e. they occur only in a given locale), but they also typically look meaningful to humans, making them suitable for a variety of geo-data visualization applications. The next section describes the data used in this work, followed by the full description of our algorithm.

\section{The Data}

Flickr has emerged as the data-source of choice for most recently developed data-driven applications in computer vision and graphics, including visual geo-location [Hays and Efros 2008; Crandall et al. 2009; Li et al. 2009]. However, the difficulty with Flickr and other consumer photo-sharing websites for geographical tasks is that there is a strong data bias towards famous landmarks. To correct for this bias and provide a more uniform sampling of the geographical space, we turn to GoOGLE STREET VIEW - a huge database of street-level imagery, captured as panoramas using specially-designed vehicles. This enables extraction of roughly fronto-parallel views of building facades and, to some extent, avoids dealing with large variations of camera viewpoint.

Given a geographical area on a map, we automatically scrape a dense sampling of panoramas of that area from Google Street View [Gronat et al. 2011]. From each panorama, we extract two perspective images (936x537 pixels), one on each side of the capturing vehicle, so that the image plane is roughly parallel to the vehicle's direction of motion. This results in approximately 10,000 images per city. For this project, we downloaded 12 cities: Paris, London, Prague, Barcelona, Milan, New York, Boston, Philadelphia, San Francisco, San Paulo, Mexico City, and Tokyo. We have also scraped suburbs of Paris for one experiment.

\section{Discovering geo-informative elements}

Our goal is to discover visual elements which are characteristic of a given geographical locale (e.g. the city of Paris). That is, we seek patterns that are both frequently occurring within the given locale, and geographically discriminative, i.e. they appear in that locale and do not appear elsewhere. Note that neither of these two requirements by itself is enough: sidewalks and cars occur frequently in Paris but are hardly discriminative, whereas the Eiffel Tower is very discriminative, but too rare to be useful $(<0.0001 \%$ in our data). In this work, we will represent visual elements by square image patches at various resolutions, and mine them from our large image database. The database will be divided into two parts: (i) the positive set containing images from the location whose visual elements we wish to discover (e.g. Paris); and (ii) the negative set containing images from the rest of the world (in our case, the other 11 cities in the dataset). We assume that many frequently occurring but uninteresting visual patterns (trees, cars, sky, etc.) will occur in both the positive and negative sets, and should be filtered out. Our 

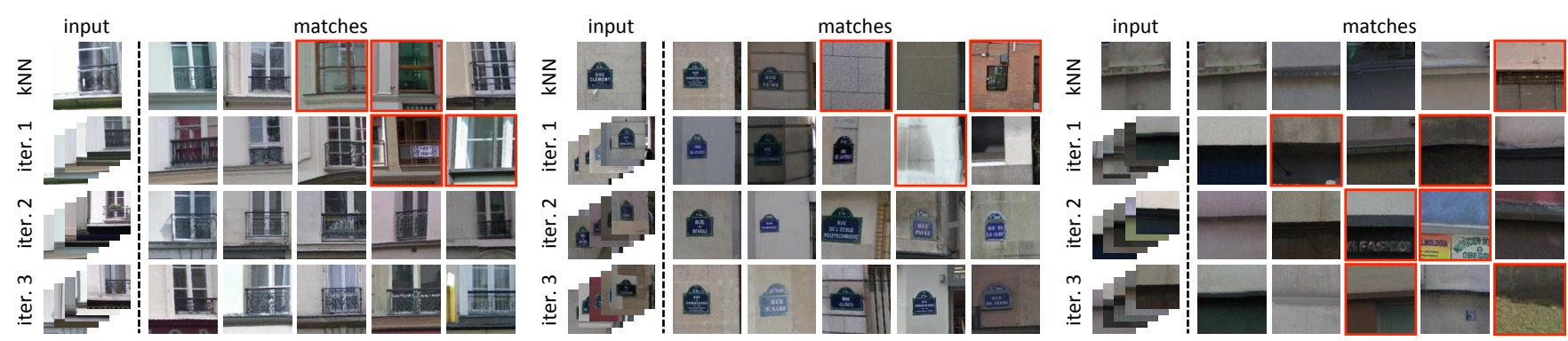

Figure 3: Steps of our algorithm for three sample candidate patches in Paris. The first row: initial candidate and its NN matches. Rows 2-4: iterations of SVM learning (trained using patches on left). Red boxes indicate matches outside Paris. Rows show every 7 th match for clarity. Notice how the number of not-Paris matches decreases with each iteration, except for rightmost cluster, which is eventually discarded.
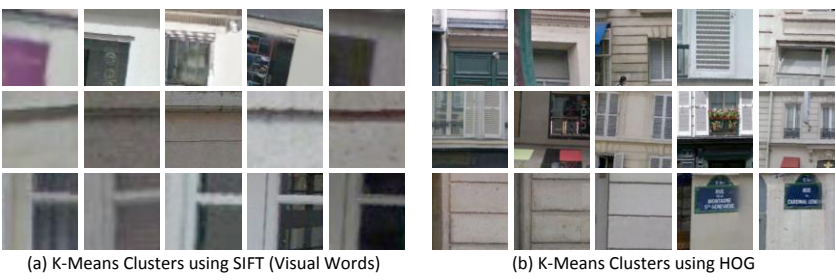

Figure 2: (a) k-means clustering using SIFT (visual words) is dominated by low level features. (b) k-means clustering over higher dimensional HOG features produces visually incoherent clusters.

biggest challenge is that the overwhelming majority of our data is uninteresting, so matching the occurrences of the rare interesting elements is like finding a few needles in a haystack.

One possible way to attack this problem would be to first discover repeated elements and then simply pick the ones which are the most geographically discriminative. A standard technique for finding repeated patterns in data is clustering. For example, in computer vision, "visual word" approaches [Sivic and Zisserman 2003] use k-means clustering on image patches represented by SIFT descriptors. Unfortunately, standard visual words tend to be dominated by low-level features, like edges and corners (Figure 2a), not the larger visual structures we are hoping to find. While we can try clustering using larger image patches (with a higher-dimensional feature descriptor, such as HOG [Dalal and Triggs 2005]), k-means behaves poorly in very high dimensions because the distance metric becomes less meaningful, producing visually inhomogeneous clusters (Figure 2b). We also experimented with other clustering approaches, such as Locality-Sensitive Hashing [Gong and Lazebnik 2011], with similar results.

An alternative approach is to use the geographic information as part of the clustering, extracting elements that are both repeated and discriminative at the same time. We have experimented with such discriminative clustering methods [Moosmann et al. 2007; Fulkerson et al. 2008; Shotton et al. 2008], but found they did not provide the right behavior for our data: they either produce inhomogeneous clusters or focus too much on the most common visual features. We believe this is because such approaches include at least one step that partitions the entire feature space. This tends to lose the needles in our haystack: the rare discriminative elements get mixed with, and overwhelmed by, less interesting patches, making it unlikely that a distinctive element could ever emerge as its own cluster.

In this paper, we propose an approach that avoids partitioning the entire feature space into clusters. Instead, we start with a large number of randomly sampled candidate patches, and then give each candidate a chance to see if it can converge to a cluster that is both frequent and discriminative. We first compute the nearest neighbors of each candidate, and reject candidates with too many neighbors in the negative set. Then we gradually build clusters by applying iterative discriminative learning to each surviving candidate. The following section presents the details of this algorithm.

\subsection{Our Approach}

From the tens of millions of patches in our full positive set, we randomly sample a subset of 25,000 high-contrast patches to serve as candidates for seeding the clusters. Throughout the algorithm, we represent such patches using a $\mathrm{HOG}+$ color descriptor [Dalal and Triggs 2005]. First, the initial geo-informativeness of each patch is estimated by finding the top 20 nearest neighbor $(\mathrm{NN})$ patches in the full dataset (both positive and negative), measured by normalized correlation. Patches portraying non-discriminative elements tend to match similar elements in both positive and negative set, while patches portraying a non-repeating element will have moreor-less random matches, also in both sets. Thus, we keep the candidate patches that have the highest proportion of their nearest neighbors in the positive set, while also rejecting near-duplicate patches (measured by spatial overlap of more than $30 \%$ between any 5 of their top 50 nearest neighbors). This reduces the number of candidates to about 1000 .

Figure 3 (top row) shows three example candidate patches together with their nearest neighbor matches. We can see that, although some matches appear reasonable, many are not very geodiscriminative (red boxes show matches outside Paris), nor visually coherent (e.g. street sign). The main problem is that a standard distance metric, such as normalized correlation, does not capture what the important parts are within an image patch, and instead treats all pixels equally. For example, the the street sign candidate (Figure 3 center), has a dark vertical bar along the right edge, and so all the retrieved NN matches also have that bar, even though it's irrelevant to the street sign concept.

Recently, [Shrivastava et al. 2011] showed how one can improve visual retrieval by adapting the distance metric to the given query using discriminative learning. We adopt similar machinery, training a linear SVM detector for each visual element in an iterative manner as in [Singh et al. 2012] while also adding a weak geographical constraint. The procedure produces a weight vector which corresponds to a new, per-element similarity measure that aims to be geodiscriminative. Our iterative clustering works as follows. Initially, we train an SVM detector for each visual element, using the top $k$ nearest neigbors from the positive set as positive examples, and all negative-set patches as negative examples. While this produces a small improvement (Figure 3 (row 2)), it is not enough, since the top $k$ matches might not have been very good to begin with. So, we iterate the SVM learning, using the top $k$ detections from previous round as positives (we set $k=5$ for all experiments). The idea is that with each round, the top detections will become better and better, resulting in a continuously improving detector. However, doing this directly would not produce much improvement because the SVM tends to overfit to the initial positive examples [Singh et al. 


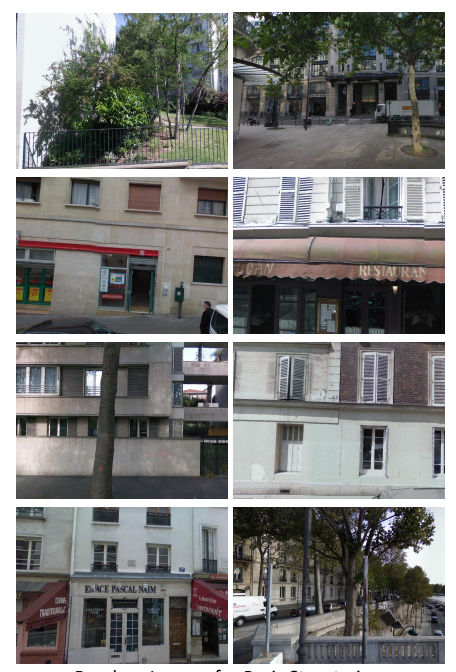

Random Images for Paris Street-view

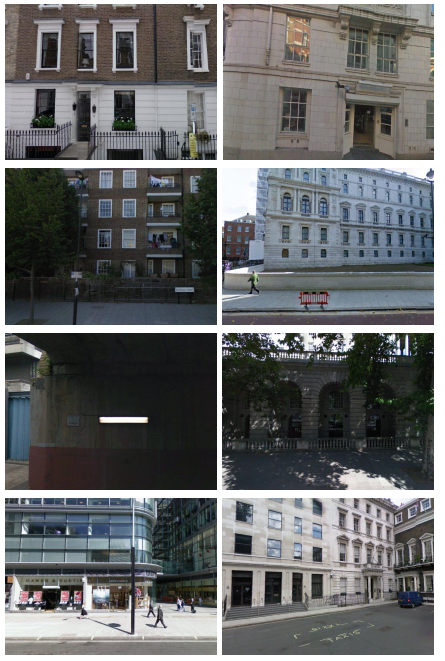

Random Images for London Street-view
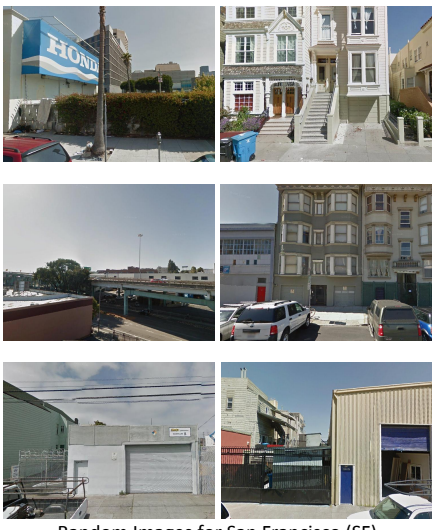

Random Images for San Francisco (SF)

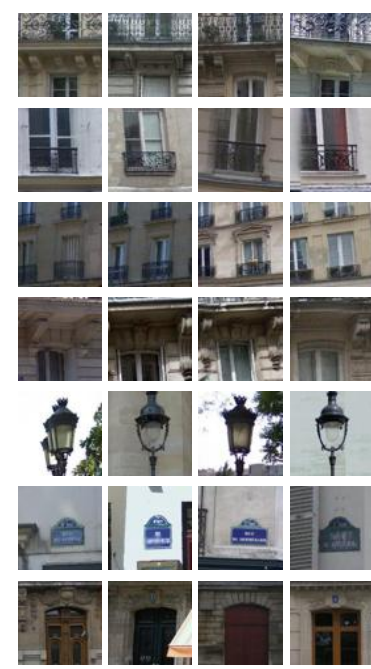

Extracted Visual Elements from Paris
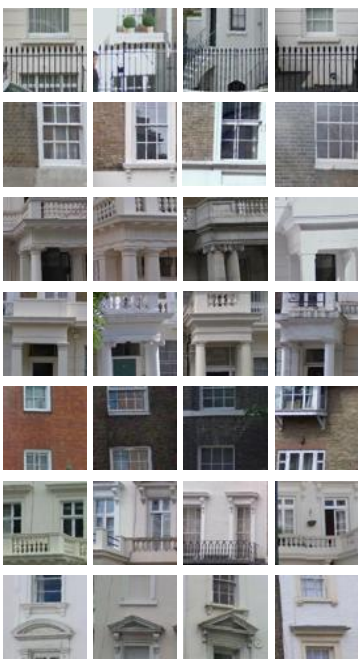

Extracted Elements from London

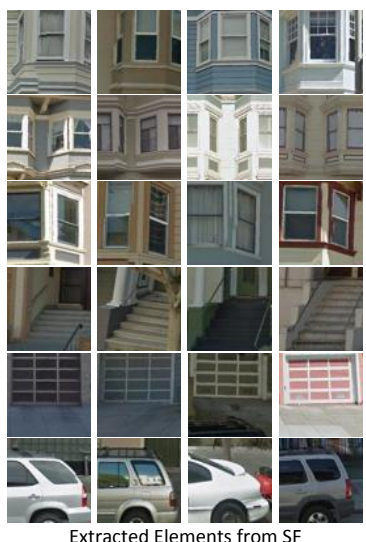

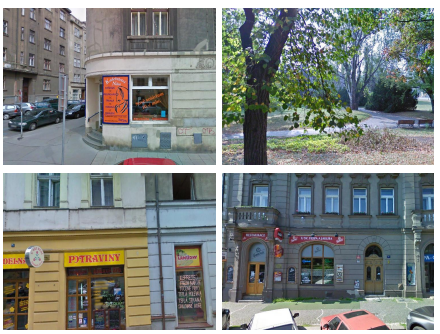
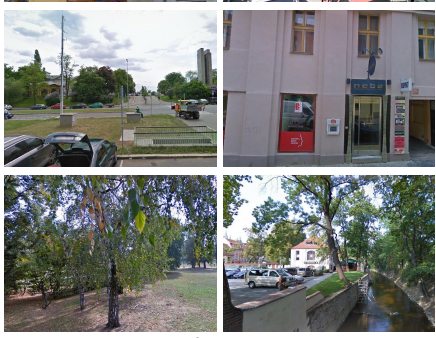

Random Images for Prague Street-view
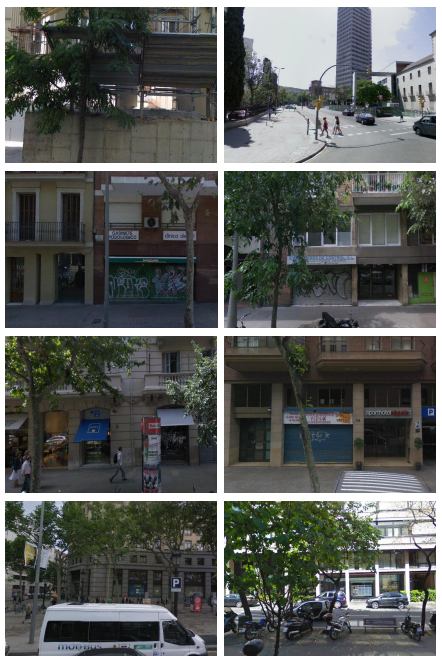

Random Images for Barcelona Street-view
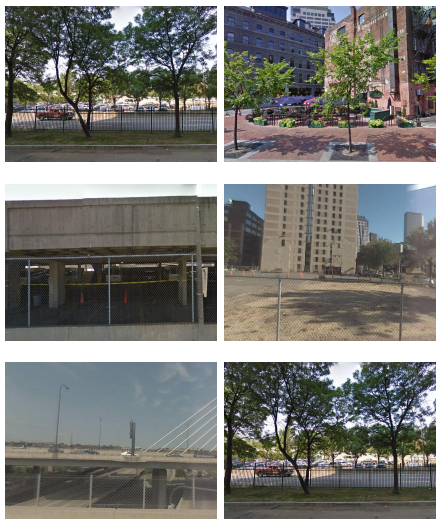

Random Images for Boston
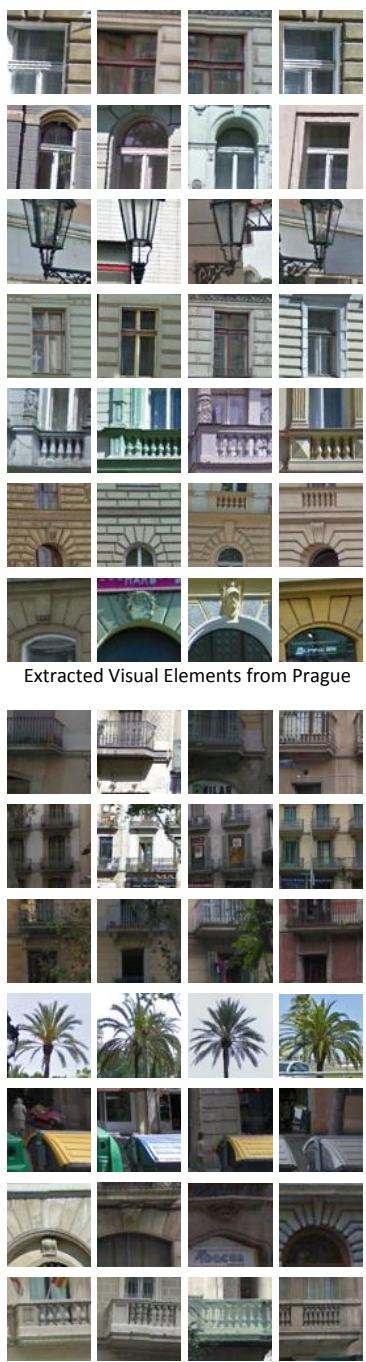

Extracted Elements from Barcelona

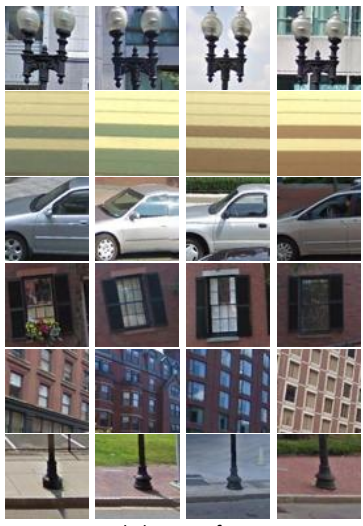

Figure 4: Google Street View vs. geo-informative elements for six cities. Arguably, the geo-informative elements (right) are able to provide better stylistic representation of a city than randomly sampled Google Street View images (left).

2012], and will prefer them in each next round over new (and better) ones. Therefore, we apply cross-validation by dividing both the positive and the negative parts of the dataset into $l$ equally-sized subsets (we set $l=3$ for all experiments). At each iteration of the training, we apply the detectors trained on the previous round to a new, unseen subset of data to select the top $k$ detections for retraining. In our experiments, we used three iterations, as most good clustered didn't need more to converge (i.e. stop changing). After the final iteration, we rank the resulting detectors based on their accuracy: percentage of top 50 firings that are in the positive dataset (i.e. in Paris). We return the top few hundred detectors as our geo-informative visual elements.

Figure 3 gives some intuition about the algorithm. For example, in the left column, the initial nearest neighbors contain only a few 
windows with railings. However, windows with railings differ more from the negative set than the windows without railings; thus the detector quickly becomes more sensitive to them as the algorithm progresses. The right-most example does not appear to improve, either in visual similarity or in geo-discriminativeness. This is because the original candidate patch was intrinsically not very geo-informative and would not make a good visual element. Such patches have a low final accuracy and are discarded.

Implementation Details: Our current implementation considers only square patches (although it would not be difficult to add other aspect ratios), and takes patches at scales ranging from 80-by-80 pixels all the way to height-of-image size. Patches are represented with standard HOG [Dalal and Triggs 2005] (8x8x31 cells), plus a $8 \times 8$ color image in $\mathrm{L}^{*} \mathrm{a} * \mathrm{~b}$ colorspace ( $\mathrm{a}$ and $\mathrm{b}$ only). Thus the resulting feature has $8 \times 8 \times 33=2112$ dimentions. During iterative learning, we use a soft-margin SVM with $C$ fixed to 0.1 . The full mining computation is quite expensive; a single city requires approximately 1,800 CPU-hours. But since the algorithm is highly parallelizable, it can be done overnight on a cluster.

\subsection{Results and Validation}

Figure 4 shows the results of running our algorithm on several wellknown cities. For each city, the left column shows randomly chosen images from Google Street View, while the right column shows some of the top-ranked visual element clusters that were automatically discovered (due to space limitations, a subset of elements was selected manually to show variety; see the project webpage for the full list). Note that for each city, our visual elements convey a better stylistic feel of the city than do the random images. For example, in Paris, the top-scoring elements zero-in on some of the main features that make Paris look like Paris: doors, balconies, windows with railings, street signs and special Parisian lampposts. It is also interesting to note that, on the whole, the algorithm had more trouble with American cities: it was able to discover only a few geo-informative elements, and some of them turned out to be different brands of cars, road tunnels, etc. This might be explained by the relative lack of stylistic coherence and uniqueness in American cities (with its melting pot of styles and influences), as well as the supreme reign of the automobile on American streets.

In addition to the qualitative results, we would also like to provide a more quantitative evaluation of our algorithm. While validating data-mining approaches is difficult in general, there are a few questions about our method that we can measure: 1) do the discovered visual elements correspond to an expert opinion of what visually characterizes a particular city? 2) are they indeed objectively geoinformative? 3) do users find them subjectively geo-informative in a visual discrimination task? and 4) can the elements be potentially useful for some practical task? To answer the first question, we consulted a respected volume on 19th century Paris architecture [Loyer 1988]. We found that a number of stylistic visual elements mentioned in the book correspond quite well to those discovered by our algorithm, as illustrated on Figure 5.

To evaluate how geo-informative our visual elements are, we ran the top 100 Paris element detectors over an unseen dataset which was $50 \%$ from Paris and 50\% from elsewhere. For each element, we found its geo-informativeness by computing the percentage of the time it fired in Paris out of the top 100 firings. The average accuracy of our top detectors was $83 \%$ (where chance is $50 \%$ ). We repeated this for our top 100 Prague detectors, and found the average accuracy on an unseen dataset of Prague to be $92 \%$. Next, we repeated the above experiment with people rather than computers. To avoid subject fatigue, we reduced the dataset to 100 visual elements, 50 from Paris and 50 from Prague. 50\% of the elements were the top-
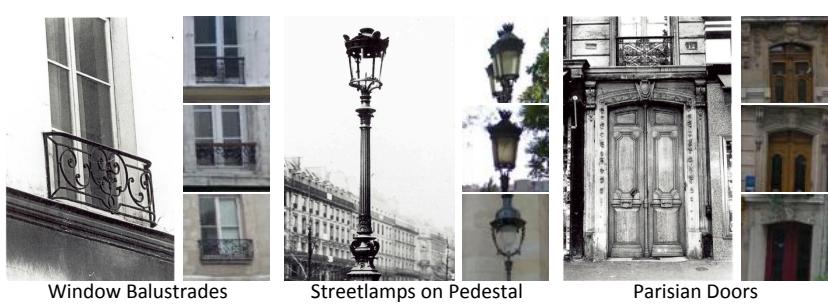

Figure 5: Books on Paris architecture are expressly written to give the reader a sample of the architectural elements that are specifically Parisian. We consulted one such volume [Loyer, 1988] and found that a number of their illustrative examples (left) were automatically discovered by our method (right).
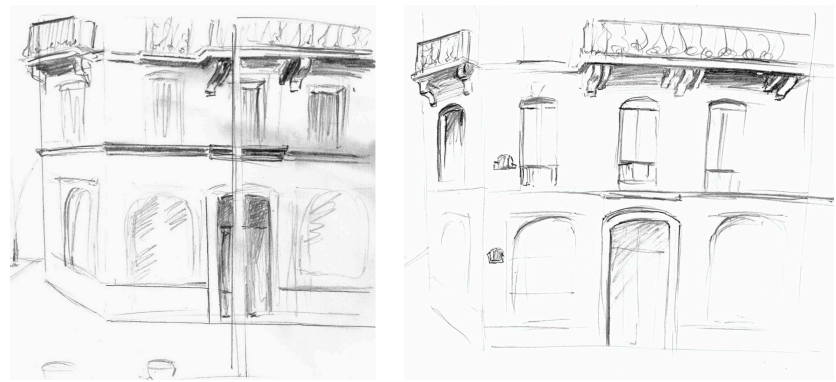

Figure 6: Geo-informative visual elements can provide subtle cues to help artists better capture the visual style of a place. We asked an artist to make a sketch from a photo of Paris (left), and then sketch it again after showing her the top discovered visual elements for this image (right). Note, for example, that the street sign and window railings are missing in the left sketch. In our informal survey, most people found the right sketch to be more Paris-like.

ranked ones returned by our algorithm for Paris and Prague. The other $50 \%$ were randomly sampled patches of Paris and Prague (but biased to be high-contrast, as before, to avoid empty sky patches, etc). In a web-based study, subjects (who have all been to Paris but not necessarily Prague) were asked to label each of the 100 patches as belonging to either Paris or Prague (forced choice). The results of our study (22 naive subjects) are as follows: average classification performance for the algorithm-selected patches was $78.5 \%$ $(s t d=11.8)$, while for random patches it was $58.1 \%(s t d=6.1)$; the $p$-value for a paired-samples $t$-test was $<10^{-8}$. While on random patches subjects did not do much better than chance, performance on our geo-informative elements was roughly comparable to the much simpler full-image classification task reported in the beginning of the paper (although since here we only used Prague, the setups are not quite the same).

Finally, to get a sense of whether our elements might serve as "reference art," we asked an artist to sketch a photograph of Paris, allowing only 10 minutes so that some details had to be omitted. Several days later, she made another 10-minute sketch of the same photograph, this time aided by a display of the top 10 geo-informative elements our algorithm detected in the image. In an informal, randomized survey, 10 out of our 11 naive subjects (who had all been to Paris) found the second sketch to be more Paris-like. The two sketches are shown in Figure 6.

\section{Applications}

Now that we have a tool for discovering geographically-informative visual elements for a given locale, we can use them to explore ways of building stylistic narratives for cities and of making visual connections between them. Here we discuss just a few such directions. 

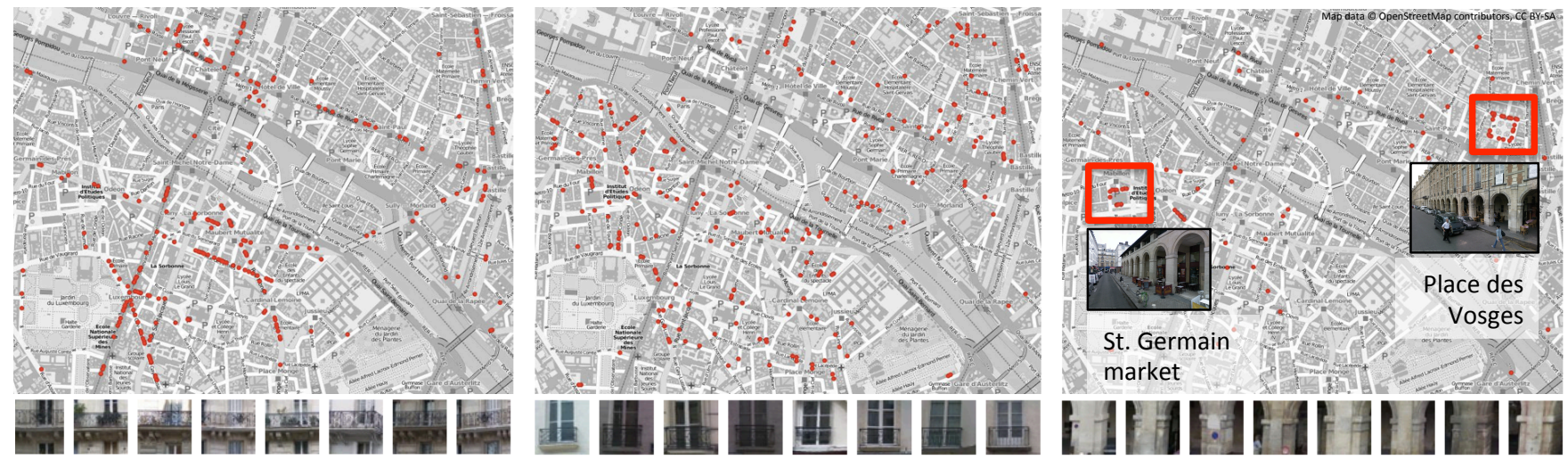

Figure 7: Examples of geographic patterns in Paris (shown as red dots on the maps) for three discovered visual elements (shown below each map). Balconies with cast-iron railings are concentrated on the main boulevards (left). Windows with railings mostly occur on smaller streets (middle). Arch supporting columns are concentrated on Place des Vosges and the St. Germain market (right).
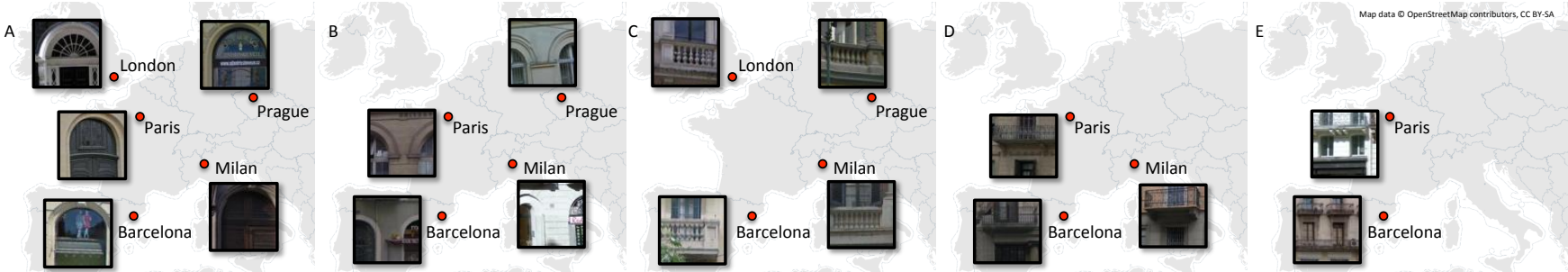

Figure 8: Architectural patterns across Europe. While arches (A) are common across all Europe, double arches $(B)$ seem rare in London. Similarly, while Paris, Barcelona and Milan all share cast-iron railings on their balconies $(D)$, the grid-like balcony arrangement (E) of Paris and Barcelona is missing in Milan.

\subsection{Mapping Patterns of Visual Elements}

So far, we have shown the discovered visual elements for a given city as an ordered list of patch clusters (Figure 4). Given that we know the GPS coordinates of each patch, however, we could easily display them on a map, and then search for interesting geo-spatial patterns in the occurrences of a given visual element. Figure 7 shows the geographical locations for the top-scoring detections for each of 3 different visual elements (a sampling of detections are shown below each map), revealing interestingly non-uniform distributions. For example, it seems that balconies with cast-iron railings (left) occur predominantly on the large thoroughfares (bd SaintMichel, bd Saint-Germain, rue de Rivoli), whereas windows with cast-iron railings (middle) appear mostly on smaller streets. The arch-supporting column (right) is a distinguishing feature of the famous Place des Vosges, yet it also appears in other parts of Paris, particularly as part of more recent Marché Saint-Germain (this is a possible example of so-called "architectural citation"). Automatically discovering such architectural patterns may be useful to both architects and urban historians.

\subsection{Exploring Different Geo-spatial Scales}

So far we have focused on extracting the visual elements which summarize appearance on one particular scale, that of a city. But what about visual patterns across larger regions, such as a continent, or a more specific region, such as a neighborhood? Here we demonstrate visual discovery at different geo-spatial scales.

We applied our algorithm to recover interesting patterns shared by the cities on the European subcontinent. Specifically, we used Street View images from five European cities (Barcelona, London, Milan, Paris and Prague) as the positive set, and the remaining 7 non-European cities as the negative set. Figure 8 shows some interesting discriminative features and patterns in terms of their membership across the 5 European cities. For example, while arches

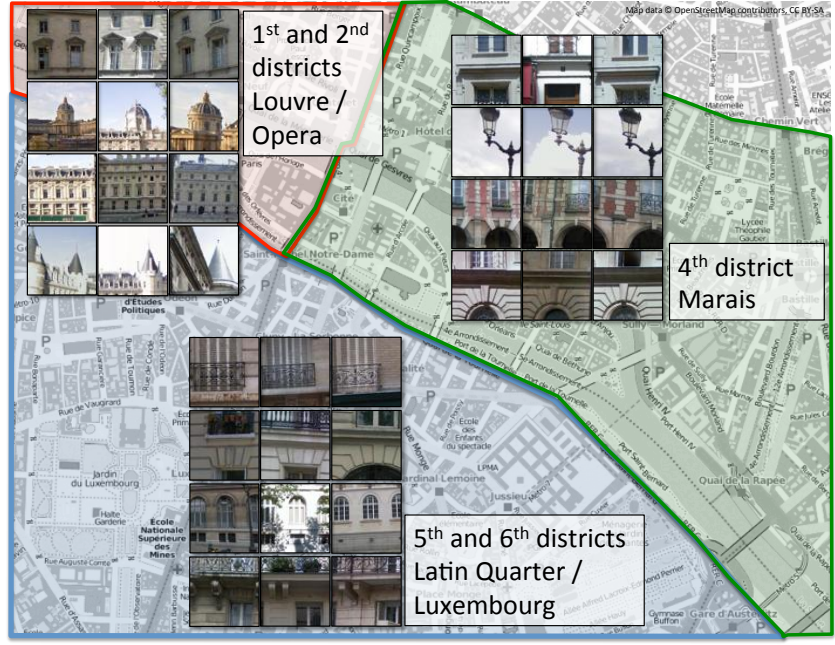

Figure 9: Geographically-informative visual elements at the scale of city neighborhoods. Here we show a few discovered elements particular to three of the central districts of Paris: Louvre/Opera, the Marais, and the Latin Quarter/Luxembourg.

are common in cities across Europe, double-arches seem rare in London. Similarly, while balcony railings in Paris, Barcelona and Milan are all made of cast iron, they tend to be made of stone in London and Prague.

We also analyzed visual patterns at the scale of a city neighborhood. Specifically, we considered three well-defined districts of Paris: Louvre/Opera (1e, 2e), Le Marais (4e), and Latin Quarter/Luxembourg $(5 \mathrm{e}, 6 \mathrm{e})$. Figure 9 shows examples of geographically informative elements for each of the three districts (while taking the other districts and Paris suburbs as the negative set). Pre- 

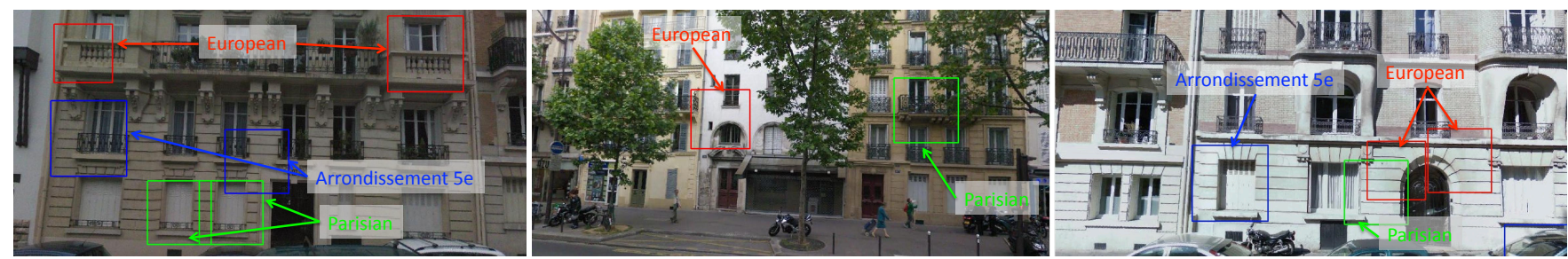

Figure 10: Detecting architectural influences. Each image shows confident detections for architectural styles at different geographic scales.

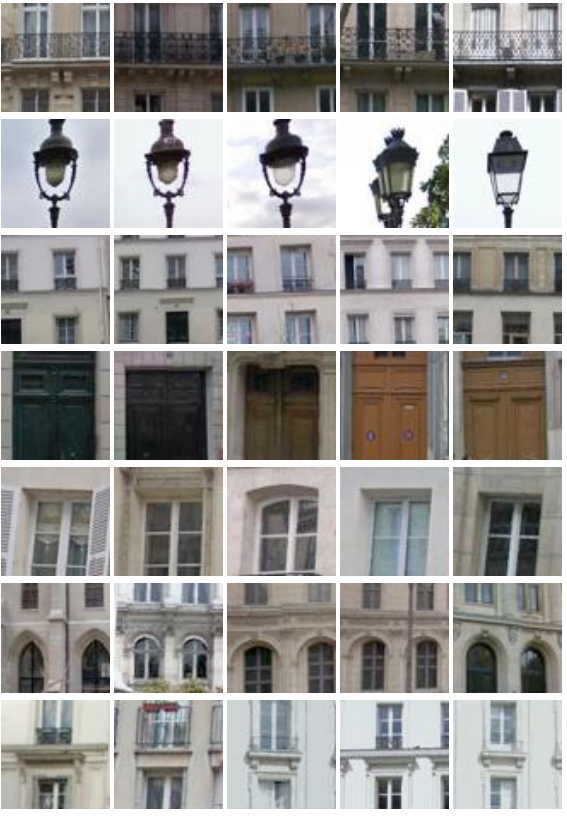

Paris, France

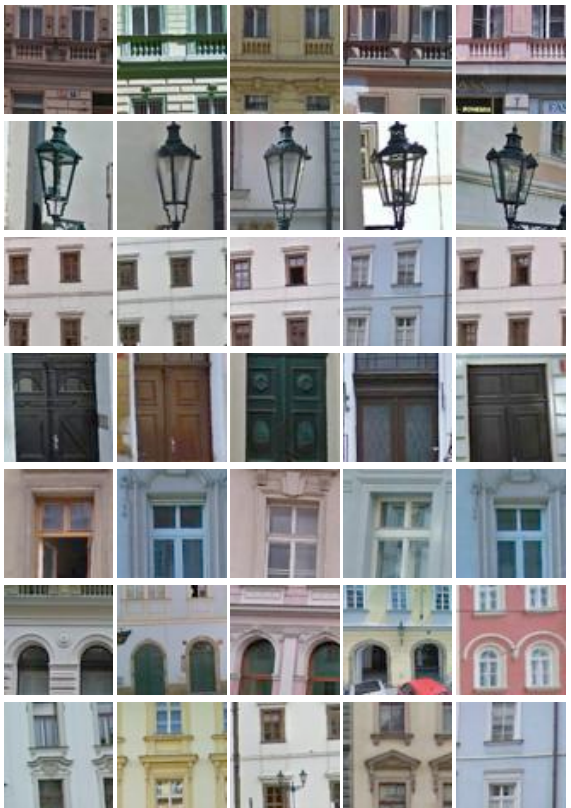

Prague, Czech Republic
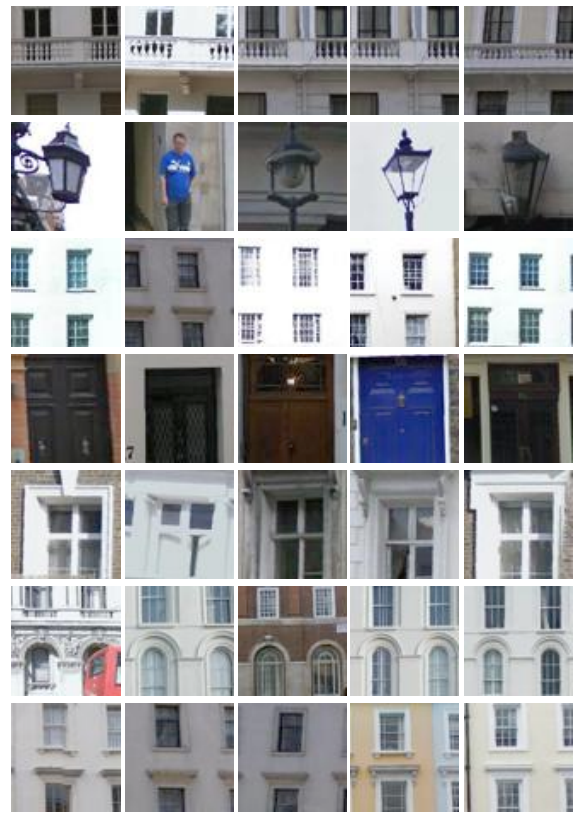

London, England

Figure 11: Visual Correspondence. Each row shows corresponding detections of a single visual element detector across three different cities.

dictably, Louvre/Opera is differentiated from the rest of Paris by the presence of big palatial facades. Le Marais is distinguished by its more cozy palaces, very close-up views due to narrow streets, and a specific shape of lampposts. Interestingly, one of the defining features of the Latin Quarter/Luxembourg is the high frequency of windows with closed shutters as compared to other districts in Paris. One possible explanation is that this neighborhood has become very prestigious and a lot of its real-estate has been bought up by people who don't actually live there most of the time.

Given the detectors for visual elements at different geo-spatial scales, it becomes possible to analyze a scene in terms of the regions from which it draws its architectural influences. Figure 10 shows images from the 5th arrondissement of Paris, pointing out which elements are specific to that arrondissement, which are Paris-specific, and which are pan-European. For example, the stone balcony railings and arches are pan-European, windows with collapsible shutters and balconies with iron railings are Parisian, and the grooves around the windows are typical of the 5th arrondissement.

\subsection{Visual Correspondences Across Cities}

Given a set of architectural elements (windows, balconies, etc.) discovered for a particular city, it is natural to ask what these same elements might look like in other cities. As it turns out, a minor modification to our algorithm can often accomplish this task. We have observed that a detector for a location-specific architectural element will often fire on functionally similar elements in other cities, just with a much lower score. That is, a Paris balcony detector will return mostly London balconies if it is forced to run only

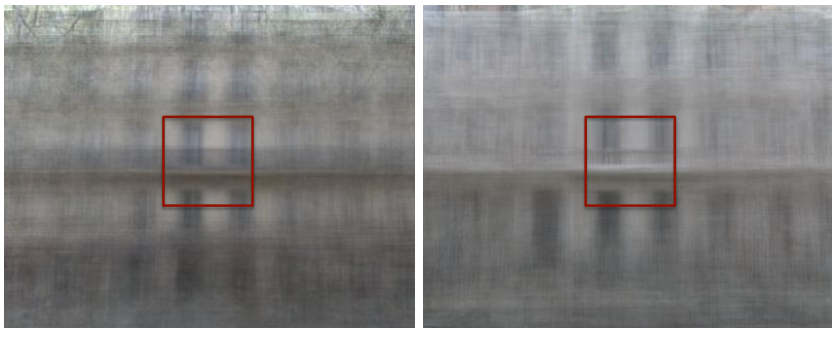

Figure 12: Object-centric image averages for the element detector in the top row of Figure 11. Note how the context captures the differences in facade styles between Paris (left) and London (right).

on London images. Naturally these results will be noisy, but we can clean them up using an iterative learning approach similar to the one in Section 4.1. The only difference is that we require the positive patches from each iteration of training to be taken not just from the source city, but from all the cities where we wish to find correspondences. For example, to find correspondences between Paris, Prague, and London, we initialize with visual elements discovered in Paris and then, at each round of "clean-up" training, we use 9 top positive matches to train each element SVM, 3 from each of the three cities. Figure 11 illustrates the result of this procedure. Note how capturing the correspondence between similar visual elements across cities can often highlight certain stylistic differences, such as the material for the balconies, the style of the street-lamps, or the presence and position of ledges on the facades.

Another interesting observation is that some discovered visual el- 

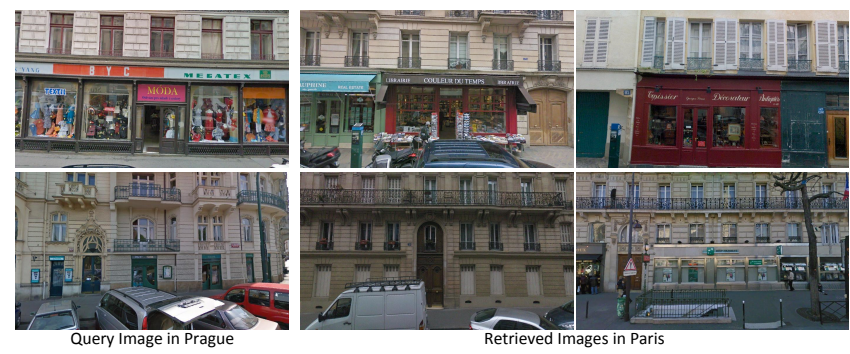

Figure 13: Geographically-informed retrieval. Given a query Prague image (left), we retrieve images in Paris (right).

ements, despite having a limited spatial extent, can often encode a much larger architectural context. This becomes particularly apparent when looking at the same visual element detector applied in different cities. Figure 12 shows object-centric averages (in the style of [Torralba and Oliva 2003]) for the detector in the top row of Figure 11 for Paris and London. That is, for each city, the images with the top 100 detections of the element are first centered on that element and then averaged together in image space. Note that not only do the average detections (red squares) look quite different between the two cities, but the average contexts reveal quite a lot about the differences in the structure and style of facades. In Paris, one can clearly see four equal-height floors, with a balcony row on the third floor. In London, though, floor heights are uneven, with the first floor much taller and more stately.

\subsection{Geographically-informed Image Retrieval}

Once we have detectors that set up the correspondence between different cities such as Paris and Prague (Sec. 5.3), we can use them for geographically-informed image retrieval. Given a query image from one location, such as Prague, our task is to retrieve similar images from another location, such as Paris. For this we use the correspondence detectors from Sec. 5.3 while also encoding their spatial positions in the image. In particular, we construct a feature vector of the query image by building a spatial pyramid and max-pooling the SVM scores of the correspondence detectors in each spatial bin in the manner of [Li et al. 2010]. Retrieval is then performed using the Euclidean distance between the feature vectors. Figure 13 demonstrates this approach where a query image from Prague retrieves images from Paris that contain similar balconies with cast iron railings (bottom) while honoring spatial layout of facades.

\section{Conclusion}

So, what makes Paris look like Paris? We argued that the "look and feel" of a city rests not so much on the few famous landmarks (e.g. the Eiffel Tower), but largely on a set of stylistic elements, the visual minutiae of daily urban life. We proposed a method that can automatically find a subset of such visual elements from a large dataset offered by Google Street View, and demonstrated some promising applications. This work is but a first step towards our ultimate goal of providing stylistic narratives to explore the diverse visual geographies of our world. Currently, the method is limited to discovering only local elements (image patches), so a logical next step would be trying to capture larger structures, both urban (e.g. facades), as well as natural (e.g. fields, rivers). Finally, the proposed algorithm is not limited to geographic data, and might potentially be useful for discovering stylistic elements in other weakly supervised settings, e.g. "What makes an Apple product?"

Acknowledgements: We thank Jessica Hodgins, Lavanya Sharan, and Yves Ubelmann for their valuable comments and suggestions. This work is a part of a larger effort with Dan Huttenlocher and
David Crandall, on modeling geo-informative visual attributes. We thank Google for letting us publish the Street View images. This work was partially supported by NDSEG fellowship to CD and by Google, NSF IIS0905402, EIT-ICT, ONR N000141010934, ONR N000141010766, and MSR-INRIA.

\section{References}

Berg, T., AND BerG, A. 2009. Finding iconic images. In The 2nd Internet Vision Workshop at Conference on Computer Vision and Pattern Recognition (CVPR).

Bourdev, L., And MaliK, J. 2009. Poselets: Body part detectors trained using 3D human pose annotations. In IEEE 12th International Conference on Computer Vision (ICCV), 1365-1372.

Chen, D., Baatz, G., Koser, K., Tsai, S., Vedantham, R., Pylvanainen, T., Roimela, K., Chen, X., Bach, J., Pollefeys, M., Girod, B., AND GrzeszczuK, R. 2011. City-scale landmark identification on mobile devices. In IEEE Conference on Computer Vision and Pattern Recognition (CVPR), 737-744.

Chum, O., Perdoch, M., and Matas, J. 2009. Geometric minhashing: Finding a (thick) needle in a haystack. In IEEE Conference on Computer Vision and Pattern Recognition (CVPR), $17-24$.

Crandall, D., Backstrom, L., Huttenlocher, D., And KLeINBerg, J. 2009. Mapping the world's photos. In Proceedings of the 18th International Conference on World Wide Web (WWW), 761-770.

DALAL, N., AND TRIGGS, B. 2005. Histograms of oriented gradients for human detection. In IEEE Conference on Computer Vision and Pattern Recognition (CVPR), vol. 1, 886-893.

Fiss, J., Agarwala, A., And Curless, B. 2011. Candid portrait selection from video. ACM Transactions on Graphics (SIGGRAPH Asia) 30, 6, 128.

Fulkerson, B., Vedaldi, A., And SoAtto, S. 2008. Localizing objects with smart dictionaries. In European Conference on Computer Vision (ECCV), 179-192.

Gong, Y., AND LAZEBNIK, S. 2011. Iterative quantization: A procrustean approach to learning binary codes. In IEEE Conference on Computer Vision and Pattern Recognition (CVPR), $817-824$.

Gronat, P., Havlena, M., Sivic, J., And Pajdla, T. 2011. Building streetview datasets for place recognition and city reconstruction. Tech. Rep. CTU-CMP-2011-16, Czech Tech Univ.

HAYS, J., AND EFros, A. 2008. Im2gps: estimating geographic information from a single image. In IEEE Conference on Computer Vision and Pattern Recognition (CVPR), 1-8.

Kalogerakis, E., Vesselova, O., Hays, J., Efros, A., And HERTZMANN, A. 2009. Image sequence geolocation with human travel priors. In IEEE 12th International Conference on Computer Vision (ICCV), 253-260.

Karlinsky, L., Dinerstein, M., And Ullman, S. 2009. Unsupervised feature optimization (ufo): Simultaneous selection of multiple features with their detection parameters. In IEEE Conference on Computer Vision and Pattern Recognition (CVPR), 1263-1270.

Knopp, J., Sivic, J., AND PAJdla, T. 2010. Avoiding confusing features in place recognition. In European Conference on Computer Vision (ECCV), 748-761. 
LeE, Y., And Grauman, K. 2009. Foreground focus: Unsupervised learning from partially matching images. International Journal of Computer Vision (IJCV) 85, 2, 143-166.

Li, X., Wu, C., ZaCh, C., LAZEBnik, S., And Frahm, J.-M. 2008. Modeling and recognition of landmark image collections using iconic scene graphs. In European Conference on Computer Vision (ECCV), 427-440.

Li, Y., Crandall, D., And Huttenlocher, D. 2009. Landmark classification in large-scale image collections. In IEEE 12th International Conference on Computer Vision (ICCV), 1957-1964.

LI, L., Su, H., Xing, E., AND FeI-FeI, L. 2010. Object bank: A high-level image representation for scene classification and semantic feature sparsification. In Advances in Neural Information Processing Systems (NIPS), vol. 24.

LOYER, F. 1988. Paris nineteenth century : architecture and urbanism, 1st american ed. Abbeville Press, New York

Moosmann, F., Triggs, B., And Jurie, F. 2007. Fast discriminative visual codebooks using randomized clustering forests. In Advances in Neural Information Processing Systems (NIPS), vol. 19.

Mueller, P., Wonka, P., Haegler, S., Ulmer, A., And VAN GoOL, L. 2006. Procedural modeling of buildings. ACM Transactions on Graphics (SIGGRAPH) 25, 3, 614-623.

Oliva, A., And Torralba, A. 2006. Building the gist of a scene: The role of global image features in recognition. Progress in brain research 155, 23-36.

PAIK, K. 2006. The Art of Ratatouille. Chronicle Books.

QuACK, T., LeIBE, B., AND VAn GoOL, L. 2008. Worldscale mining of objects and events from community photo collections. In Proceedings of the International Conference on Content-based Image and Video Retrieval (CIVR), 47-56.

Russell, B. C., Efros, A. A., Sivic, J., Freeman, W. T., AND ZISSERMAN, A. 2006. Using multiple segmentations to discover objects and their extent in image collections. In IEEE Conference on Computer Vision and Pattern Recognition (CVPR), 1605-1614.

Schindler, G., Brown, M., And Szeliski, R. 2007. Cityscale location recognition. In IEEE Conference on Computer Vision and Pattern Recognition (CVPR), 1-7.

Shotton, J., Johnson, M., And Cipolla, R. 2008. Semantic texton forests for image categorization and segmentation. In IEEE Conference on Computer Vision and Pattern Recognition (CVPR), 1-8.

Shrivastava, A., Malisiewicz, T., Gupta, A., And Efros, A. A. 2011. Data-driven visual similarity for cross-domain image matching. ACM Transactions on Graphics (SIGGRAPH Asia) 30, 6, 154 .

Simon, I., SnaVely, N., AND SEITZ, S. M. 2007. Scene summarization for online image collections. In IEEE 11th International Conference on Computer Vision (ICCV), 1-8.

Singh, S., Gupta, A., And Efros, A. A. 2012. Unsupervised discovery of mid-level discriminative patches. arXiv:1205.3137 [CS.CV].

Sivic, J., AND Zisserman, A. 2003. Video google: A text retrieval approach to object matching in videos. In IEEE 9th International Conference on Computer Vision (ICCV), 1470-1477.
Sutcliffe, A. 1996. Paris: an architectural history. Yale University Press.

Teboul, O., Simon, L., Koutsourakis, P., And Paragios, N. 2010. Segmentation of building facades using procedural shape priors. In IEEE Conference on Computer Vision and Pattern Recognition (CVPR), 3105-3112.

Torralba, A., AND Oliva, A. 2003. Statistics of natural image categories. Network: Computation in Neural Systems, 391-412.

ZHENG, Y.-T., ZHAO, M., SONG, Y., AdAM, H., BUDDEMEIER, U., Bissacco, A., Brucher, F., Chua, T.-S., AND Neven, H. 2009. Tour the world: building a web-scale landmark recognition engine. In IEEE Conference on Computer Vision and Pattern Recognition (CVPR), 1085-1092. 